\title{
Demonstrating demand for preventive health services in rural Kenya
}

\author{
Jeffrey D. Lackey, Stephen Suffian, Rachel Dzombak, Khanjan Mehta* \\ Humanitarian Engineering and Social Entrepreneurship Program (HESE) \\ The Pennsylvania State University, University Park, PA, USA \\ *khanjan@engr.psu.edu
}

\begin{abstract}
Developing countries like Kenya suffer from a severe lack of healthcare professionals. Access to primary care is further limited when large distances must be travelled for basic medical care, and often times the decision to seek professional help is made too late. Preventive health services can save lives by diagnosing serious medical conditions early and subsequently reducing the likelihood of life-threatening diseases. The rapid increase in the global incidence of chronic diseases like diabetes, hypertension, and heart disease makes preventive health services particularly important. Trained community health workers (CHWs) operated the Mashavu telemedicine system in Nyeri County of Kenya over the course of five months. The Mashavu system comprised of a netbook computer, a web camera, and other basic supplies. CHWs provided patients with two alternative services: (1) body measurements including blood pressure, weight, and body mass index (BMI) or (2) a full consultation that supplemented the measurements with health indicators and a brief survey. A lightweight website was developed to allow $\mathrm{CHWs}$ to send this information to a nearby nurse. This website was accessed by leveraging the $3 G$ cell networks that are ubiquitous in Kenya. During the five-month pilot, 777 patients paid for these services. Nearly one-third of patients used the service as an opportunity for a routine health check. The median age among patients was 40 years and $35 \%$ of all patients were between the ages of 21-35. The pilot described in this paper demonstrated to CHWs that community members value health information and are willing to pay a small fee for it. This pilot suggests that as developing countries struggle to address the double burden of chronic and infectious disease, fee-for-service preventive health services can serve as an innovative means to achieve economic development and improved community health.
\end{abstract}

KEYWORDS: community health worker (CHW), preventive healthcare, telemedicine, Kenya.

1

\section{INTRODUCTION}

More than $78 \%$ of the 40 million people living in Kenya reside in rural areas (World Health Organization, 2009). However, the majority of healthcare professionals only practise in urban areas, forcing Kenyans to travel long distances to access treatment (Castro-Leal et al, 2000) (World Health Organization, 2011). The cost of transportation to and from a health facility can amount to almost a day's income, excluding income lost by missed work (Wynchak \& Fortuin, 2012; Wachira \& Martin, 2011). Often the cost of care itself leads individuals to delay treatment until a health problem interferes with daily activities. Preventive healthcare programs can save lives by diagnosing serious medical conditions early and subsequently reduce the likelihood of life-threatening diseases ( $\mathrm{Gu}$ et al, 2010). When individuals wait for a problem to become critical before seeking treatment, they have increased chances of developing a chronic condition, incurring higher treatment costs, and receiving an overall poorer prognosis (Chuma et al, 2007; Wang et al, 2005; Bolin, 1996). However, there are few instances of economically successful preventive healthcare services that are paid for by customers (Chen et al, 2013).

Mashavu is a telemedicine system that connects patients with healthcare providers with the goal of facilitating preventive healthcare in rural geographically-dispersed communities. The portal was developed by the Humanitarian Engineering and Social Entrepreneurship (HESE) Program at Penn State in collaboration with several Kenya organisations including the Children and Youth Empowerment Centre (CYEC), the Ministry of Health, and the United Nations Industrial Development Organisation (UNIDO). Three years of validation work by the authors preceded the implementation of Mashavu. The system provides economic and social value to rural Kenyans by establishing a remote connection via the Internet between patients and local medical professionals 
(Qin et al, 2012; Fleishman et al, 2010). Through decreased barriers to healthcare, such as lower cost to obtain personal health information and greater service accessibility (Ensor \& Cooper, 2004), the system alerts individuals to previously unidentified ailments and increases awareness of changes in personal health.

The Mashavu system serves as a reliable storeand-forward telemedicine system operated by Community Health Workers (CHWs). The system's intraobserver reliability rate of $90 \%$ is comparable to telemedicine systems in the western world (Qin et al, 2012). The Mashavu business plan incorporates influential community members such as CHWs, Sub-Chiefs, doctors, and elders. The most important resource for the Mashavu endeavour is the locallyresiding, unpaid volunteer network of CHWs. $\mathrm{CHWs}$ are geographically and culturally closer to the people they serve and are available when health facilities are closed (Kelly et al, 2001). Primarily, CHWs contribute their expertise by educating locals on health issues and encouraging lifestyle changes. Ultimately, CHWs' utilisation of the Mashavu system as an income-generating tool demonstrates the system's potential to increase healthcare access in rural Kenyan communities.

Evidence shows that successful treatment of some health problems is more likely if diagnosis occurs at an early stage ( $\mathrm{Gu}$ et al, 2010). The system aims to catalyse the start of treatment for any given ailment. The purposes of this study are to both examine the system's efficacy as a component of healthcare infrastructure as well as demonstrate demand for a paid preventive health service system that has the potential to evolve into a sustainable entrepreneurial venture for CHWs. This evaluation determines whether the pilot implementation of the described system effectively served key health demographics such as women and rurally based labourers. Furthermore, the fulfilment of responsibilities by CHWs and a continued flow of patients justify pursuit of establishing a sustainable version of the service. The desired model would allow for CHWs to offer greater services to their community while simultaneously earning money. The ability to generate income enables $\mathrm{CHWs}$ to devote greater amounts of time to monitoring the health of their communities.

To facilitate this study, a five-month pilot was conducted from July to December 2011 in five communities within Nyeri Constituency, Nyeri County, Kenya. This paper will first outline the implementation and operation of the Mashavu kiosks and present the data from the five-month pilot carried out by trained CHWs in Nyeri County, Kenya. This is followed by the discussion of results and will conclude with insights regarding the evolution of operations after the pilot was completed.

\section{METHODS}

\subsection{Pilot launch preparation}

The Kenyan Ministry of Public Health and Sanitation (MOPHS) identified CHWs with strong communication skills to be trained as Mashavu Health Workers (MHWs). The three CHWs selected were women, merely as a consequence of female CHWs' predominance. The Kikuyu tribe populates Nyeri County, thus MHWs often interacted with patients entirely in the native Kikuyu language. The ability of MHWs to speak in the mother tongue enabled wider system usage and the establishment of trust between the MHW and the patient. However, all information to be viewed by the nurse was entered into the computer by the MHWs in English. The authors chose to have the portal in English to ensure robustness and relevancy for later scaling.

The system operated in communities that were chosen in conjunction with the District Medical Officer of Health (DMOH) in Nyeri South District. A community's selection was influenced by its proximity to a health facility and population density. Additionally, the $\mathrm{DMOH}$ viewed the system as an opportunity to collect data from communities where no information had previously been captured. Relationships with the partner communities were first established by liaising with the Office of Public Health in Nyeri Town. These interactions led to meetings with Sub-Chiefs, village elders, local business owners and CHWs in the partner communities.

Sub-Chiefs and local business owners proved essential in crafting and organising the logistics of starting a kiosk in a particular village, as well as generating community involvement. The Sub-Chief of the villages organised a baraza, or community meeting, to gather locals and create a forum where the kiosks could be discussed. At the baraza, community members asked questions and interacted with the authors as well as the CHWs that were to operate the kiosks on a day-to-day basis. Subsequently, agreements were made with the owner of each kiosk location. Each kiosk had access to electricity, was publicly accessible, and was partitioned for an appropriate level of privacy given the personal nature of the interaction. Some locations donated the electricity usage, while others requested a small monthly fee.

Housing Mashavu kiosks proved symbiotic for businesses and offices attempting to gain greater community exposure, as the kiosks brought traffic to previously unnoticed entities. For example, the establishment of a kiosk at the Thunguma police post led community members to have greater interaction with the officers stationed at the local facility and increased familiarity with police services. Moreover, kiosks housed at government offices, such as those for the community Sub-Chief in Gatitu, increased 
the public's engagement with influential community figures and organisations.

Once the phase of community organising completed, focus shifted to training the identified CHWs and preparing them to run the kiosks independently. All three CHWs participated in MHW training without compensation over a two-week period. If any chose to no longer participate, they would have received free computer training that could be utilised to acquire other jobs. Most members of the community had minimal experience using computers due to a lack of education and access. This dictated that training be conducted with the assumption that $\mathrm{CHWs}$ had no prior computer knowledge. Standardised training included lessons on general computer usage, Microsoft Word and Excel, typing practice, Safaricom 3G modem installation and use, internet browsing, email, and usage of the Mashavu web portal. During training, the CHWs worked with the authors and progressed from observing, to collecting data with medical devices, to finally achieving full autonomy and entering all information into the computer system. Additionally during the two-week period, a nurse was trained to provide appropriate feedback that clearly indicated actions to be undertaken by the patient when warranted.

\subsection{Pilot operation}

The five-month pilot business plan was designed as a step towards the long-term sustainability of the Mashavu system as an income-generating program for CHWs. While the pilot relied heavily on investment by Mashavu, the intention was for the system to grow towards a financially stable model. In this pilot, the MHWs and nurse were both salaried as well as given all necessary equipment for operations. Furthermore, the MHWs were provided an incentive payment for each patient seen. This structure put the financial risk on Mashavu, ensuring MHWs were properly compensated for their time regardless of initial demand for the service. Each $\mathrm{MHW}$ was paid $225 \mathrm{KSH}$ (\$2.80) per day when working at a kiosk. MHWs then charged $10 \mathrm{KSH}(\$ 0.12)$ per patient interaction and received an additional incentive from Mashavu of $10 \mathrm{KSH}(\$ 0.12)$ per patient interaction. The price point of $10 \mathrm{KSH}$ for patients was decided upon by the MHWs as an appropriate amount for rural areas. To put this into perspective, a single bottle of Coca Cola, available even in the most remote shops, cost approximately $40 \mathrm{KSH}(\$ 0.50)$.

After completing training, MHWs received the resources needed to provide a patient with their body measurements or perform a full consultation. The MHW's kit included a netbook computer, internet modem, sphygmomanometer, tape measure, weighing scale, thermometer, notebook, pen, surgical spirits, tissue, white lab coats, nametag, hand sanitiser, scissors, tape, advertising flyers, and cards for information to be written and given to each patient. Batteries were initially provided for the sphygmomanometer, with an agreement that MHWs would pay for future battery purchases. Internet modems, a common USB device in Kenya that utilises both 3G and EDGE cell phone networks to provide internet access, were given to the MHWs as well. The IMT-2000 Standard defines 3G as having approximately $2 \mathrm{Mbits} /$ second for stationary users (Pandya et al, 1997). Currently, a cellular network is available in $40 \%$ of Kenya, covering areas where $95 \%$ of people live (GSMA, 2012). USB extension cables were used to move the internet modem close to a window or door, improving internet connectivity. An external Logitech webcam was also attached to a USB port. The webcam was used instead of the netbook's internal webcam so that pictures could more easily be taken, specifically of medically relevant skin rashes.

A website was built for MHWs to store patient information and review nurse responses. The website also enables the nurse to view patient information and append responses. The front-end of the website was built to be lightweight, in order to minimise loading time and the cost of data transmission. HTML, Javascript, Adobe Flash, and PHP were utilised to create an efficient user experience, with the backend storing data on a secure remote server in a mySQL database. Javascript was used to create an interactive web experience for the MHWs; Adobe Flash was used in order to connect the computer's webcam directly into the browser; PHP was used for communicating the data entered by MHWs into the remote database; mySQL was utilised to reliably store information and relay data back to the website through PHP requests.

From Monday through Friday, a MHW travelled to an advertised location to operate a kiosk from 9:00 AM to 5:00 PM. Another Mashavu kiosk provided services one day per week in each of the following areas within Nyeri County: Gatitu, Micha, Ruring'u, Thunguma, and Nyeri Town. Figure 1 shows the locations of these kiosks within Nyeri County. The locations spanned across multiple wards within Nyeri Town Constituency, and were reachable by CHWs using public transport. A nurse stationed at a central location in Nyeri County worked full-time during the course of the pilot in order to provide consultation responses. A photograph documenting the kiosk at the Thunguma Police Post can be seen in Figure 2.

When a new patient arrived for the service, the MHW created a profile for them detailing their medical history and demographic information. A screen shot from the profile creation page on the Mashavu web portal can be visualised in Appendix A: Demographic Information Screenshot. All information captured in the profile would be updated upon subsequent visits only if any significant changes had occurred.

The MHW then conducted a consultation, which included asking a series of questions about an 

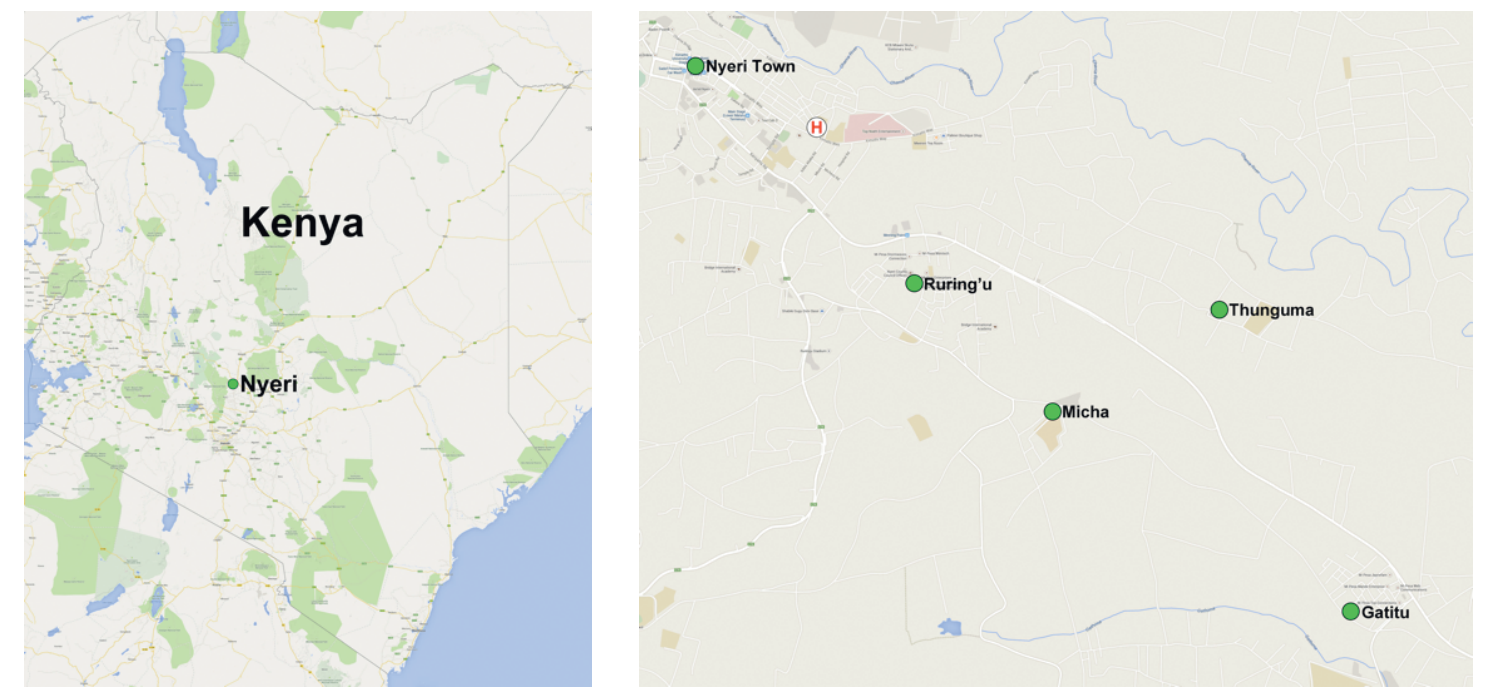

Figure 1: $\quad$ Map of kiosks (Google Maps, 2014).

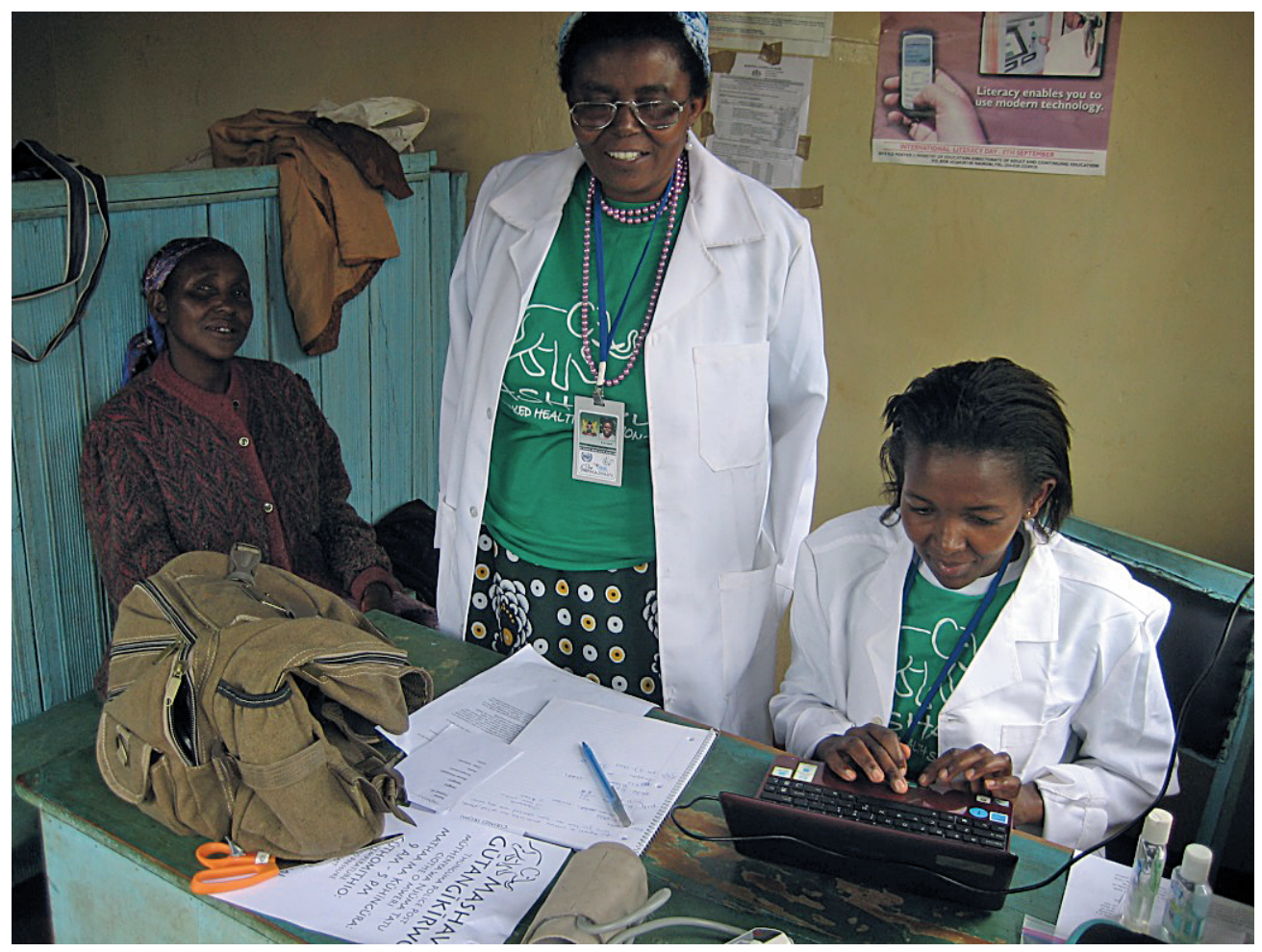

Figure 2: Mashavu kiosk during operation.

individual's health. MHWs started each consultation by asking patients to cite their "Chief Complaint" or most prevalent issue. This is captured in Appendix B: Consultation Questions Screenshot. Once complete, the MHW asked Yes/No consultation questions that were organised into seven sections: Ears/Eyes/Nose/ Throat (EENT), Heart/Lungs, Gastro-Intestinal (GI), Head, Bones/Joints, Other and For Women Only.

If a patient answered "Yes" to a question, the MHWs asked follow-up questions. For example, if someone answered "Yes" to "Do you have eye pain?" the MHW would ask, "When did the problem start?" along with other pertinent questions. Follow-up responses would be entered into a text box located below each question. The MHW also collected patients' body measurements and entered the information into the system.

Each consultation took approximately 20 minutes. Once complete, the MHW saved the consultation on a secure web portal. The nurse, stationed at a central location with electricity and internet service, logged in and accessed the newly stored information. Mashavu required the nurse to respond within 20 minutes and patients could choose to wait for their response or return to the kiosk at a more convenient time. The nurse responded to each consultation with an answer to the following question: Does the patient's health profile and body measurements warrant a trip to the nearest health facility? The nurse also provided suggestions on how the patient 


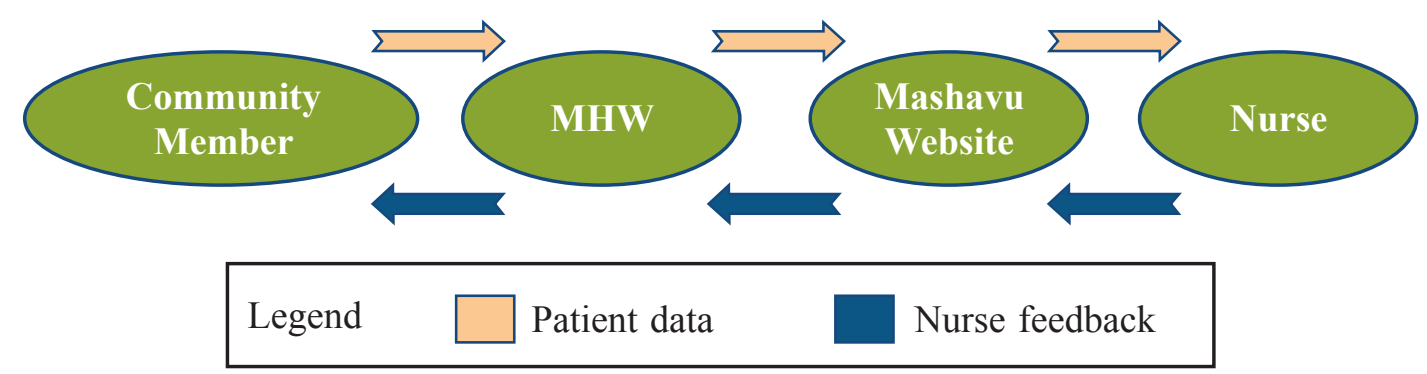

Figure 3: Flow of information.

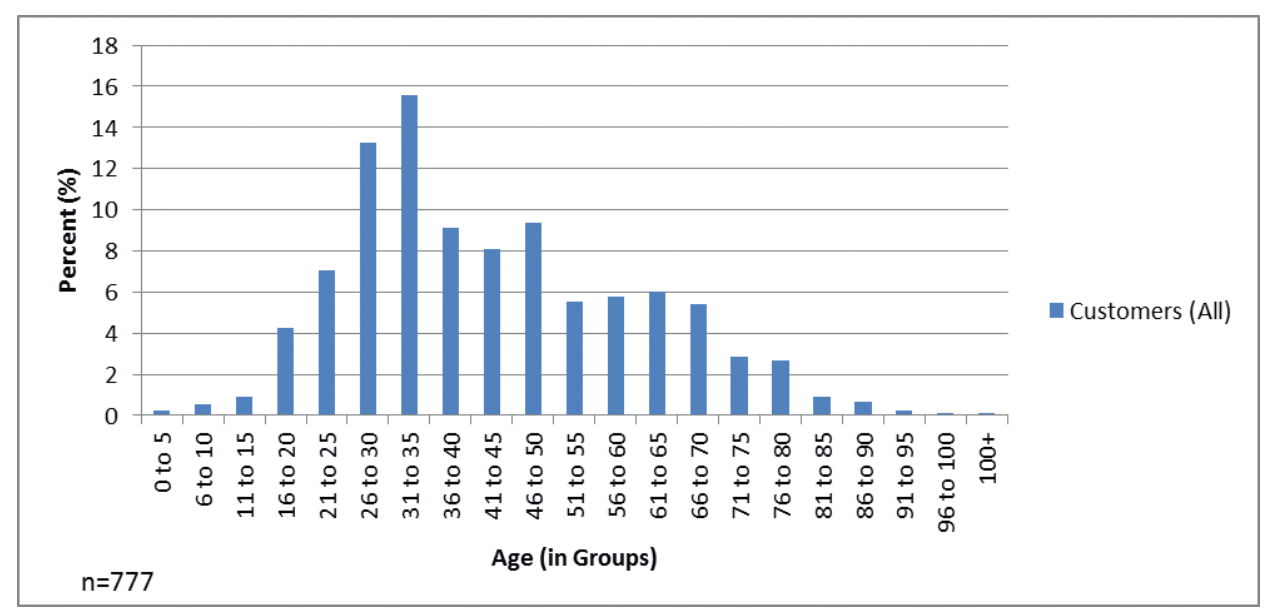

Figure 4: Age breakdown of Mashavu patients (all).

could improve their health through lifestyle changes or suggested over-the-counter remedies for their noted symptoms. No prescriptions were given and no diagnoses were made. The MHW was responsible for discussing the nurse's response with the patient and ensuring that all feedback was fully understood. The MHW used prior health training to interpret the feedback and properly explain the related medical concepts to the patient. This additional level of discussion was important for patients to comprehend and receive the full benefit of the nurse's feedback. The overall flow of information is summarised in Figure 3.

Prior to data collection the Institutional Review Board at The Pennsylvania State University approved all the research plans. Permissions were also sought from the local health authorities. All studies conducted were classified as "Exempt".

\section{RESULTS}

Over the course of five months, 777 people in Nyeri County paid for preventive health services. More than $88 \%$ of patients stated that they lived in rural communities. The most prevalent occupation among patients was "farmer" at 31\%, followed by "business person" at $26 \%$, and "student" at $7 \%$. Out of the 243 people who listed themselves as farmers, over 95\% resided in rural locations.
The largest age group among patients was between 31-35 as shown in Figure 4, and over 19\% of patients reported to be over the age of 61 , effectively skewing the unimodal age distribution to the right. Approximately $74 \%$ of patients were between the ages of 21 and 60 . Ages were voluntarily given by patients, with most lacking proper identification and unsure of their actual birth year. The overall clientele was $66 \%$ female and $34 \%$ male.

At the start of each consultation, the MHW asked the patient to state their Chief Complaint and over half of the patients responded. These complaints

Table 1: Top complaints of Mashavu patients (all).

\begin{tabular}{|c|c|}
\hline Top Complaints & Number of Patients \\
\hline Joint Pain & 126 \\
\hline EENT & 89 \\
\hline Headache & 69 \\
\hline GI & 63 \\
\hline Chest Pain & 37 \\
\hline Vision & 29 \\
\hline Gynecological & 23 \\
\hline Skin Rash & 22 \\
\hline Numbness & 18 \\
\hline
\end{tabular}


Table 2: $\quad$ Top complaints of Mashavu patients (ages 21-35).

\begin{tabular}{|c|c|}
\hline Top Complaints & Number of Patients \\
\hline EENT & 30 \\
\hline Headache & 25 \\
\hline Gl & 23 \\
\hline Joint Pain & 18 \\
\hline Gynaecological & 12 \\
\hline Vision & 10 \\
\hline Chest Pain & 9 \\
\hline Dental & 7 \\
\hline Skin Rash & 6 \\
\hline
\end{tabular}

were subsequently grouped into the set of categories displayed in Table 1, and were examined in more detail for the 21-35 age group in Table 2. Patients complained of EENT problems, including congestion, colds, cough, ear pain, dizziness, and fever. GI problems included abdominal pain, upset stomach, bloating, heartburn, constipation and loss of bladder control.

While the chief complaints conveyed reasons for using the system, greater information could be ascertained about individuals' health when the MHWs asked follow-up consultation questions. Nearly $18 \%$ of those that came to the kiosk without a chief complaint were recommended to visit a doctor at a clinic, dispensary, or hospital. High blood pressure led the nurse to recommend further care for $56 \%$ of such patients. For the population aged 21-35 years and without a chief complaint, this was true in $72 \%$ of the cases. Overall, $31 \%$ of patients exhibited signs of hypertension and $93 \%$ of them did not previously know their blood pressure was an issue.

Patients exhibiting signs of hypertension were referred to a health facility at a high rate, thus showing the ability of preventive health services to identify easily detectable, but previously unidentified, noncommunicable diseases (NCDs). Over the past three decades, the mean systolic blood pressure of Kenyans has increased, and cardiovascular disease was deemed the cause of death in $13 \%$ of autopsy studies, accounting for $25 \%$ of all hospital admissions (Yonga, 2010). With the rise of NCDs in Kenya, preventive health services can alert individuals to their risk of acquiring an NCD or, if already diagnosed, can provide a means of treatment management.

\section{DISCUSSION}

After the completion of the 5-month pilot, the results were analysed and a meeting was held with the $\mathrm{DMOH}$ to discuss the findings. Sub-Chiefs and local business owners were also periodically updated on the progress of the kiosks and notified of any system changes. Additionally, routine meetings with the
MHWs were held to discuss issues and strategies to attract more patients. During meetings, the MHWs were also notified of current trends in the data to enhance their health awareness and to supplement health knowledge.

The kiosk operated during standard work hours (9:00am-5:00 pm), which prevented a portion of the population from attending the kiosks regardless of desire. Furthermore, some patients only wanted their body measurements and did not have time to undergo a full consultation. To attract more patients, the MHWs travelled from home to home within a community during the afternoons. This change in operations that was autonomously pioneered by the MHWs during the pilot increased the convenience of the service and allowed the MHWs to better understand the conditions under which a patient lived, yielding greater insight into the symptoms they were experiencing. The MHW was then better prepared to interpret medical advice from the nurse and discuss contextually-specific implications with the patient. Interactions such as these offer far greater benefits than those from a cursory internet search that a patient could do at a cyber café in Nyeri town. Further, even simple queries are difficult for community members whose primary language is Kikuyu, a tribal language not widely spoken outside the region where the pilot took place.

Mashavu appealed to diverse populations of people within the community. Farming employed the majority of patients. While farming is a common profession amongst the general population, particularly in Kenya's Central Province, the difference in working hours between farmers and those involved in business may have led to community misrepresentation. A wide range of ages attended the kiosks and patients were most commonly women. Preventive health services provide a low-barrier opportunity for women of reproductive age to take an active interest in their health. This is especially important for young women; they are more likely to use a skilled professional during childbirth if they initiate antenatal care early in their pregnancy term (Ochako et al, 2011). Further, preventive health services have the potential to improve farmer health, thus increasing agricultural productivity and contributing to greater food security (Food and Agriculture Organization, 1996). Both farmers and young women not only sought preventive health services, but also were willing to pay a fee for access to health information.

CHWs are unpaid volunteers with limited resources for monitoring the health of their communities, and specifically the high-risk populations within them. The Mashavu system enhanced CHWs' ability to track community health and increased their health knowledge through participation in Mashavu training sessions, as well as enabled them to generate income while working as MHWs. An extensive review of 
the income generated by the MHWs throughout the pilot was published at the Humanitarian Technology: Science, Systems and Global Impact Conference in 2014 (Holmes et al, 2014). All training materials, such as those for the usage of the medical devices, were developed in a way that leveraged CHWs existing health knowledge. Basic computer training was also standardised by Mashavu and was used to train several other interested community members. With Mashavu, CHWs were also given the opportunity to generate a livelihood, thus incentivising them to help more people than they would have otherwise. The prevalence of CHWs in Kenya ensured the existence of a consistent applicant pool when scaling the program in new communities.

The system gathered health information from communities where data had never previously been collected by the Kenyan MOPHS due to a lack of facilities in the targeted rural areas. This lack of information consequently prevents the MOPHS from taking any decisive actions to address issues. Additionally, it inhibits the government from validating the effectiveness and the impact of any existing health initiatives. Mashavu enabled CHWs to spend time gathering critical data about the health status of communities. It effectively extended the reach of the MOPHS well beyond the existing infrastructure's capacity by generating extensive information on the residents' health history. This provided an opportunity for the MOPHS to make a community diagnosis and undertake targeted preventive health campaigns, reducing the future burden on already sparse health facilities due to untreated conditions.

Mashavu's implementation strategy and effectiveness at generating health awareness gave rise to the program's applicability in other communities and addressed the resource problems that exist in the MOPHS. In 2000, the MOPHS implemented a Resource Allocation Criteria (RAC) formula to address the need for a transparent, objective, efficient, and equitable resource distribution process, but the poor quality of data utilised in the RAC formula hindered the potential for impact (Briscombe et al, 2010). The formula can be improved through greater data collection on a community level. The system can obtain such information and simultaneously help specified locations to better lobby for funds and create defined and executable health initiatives.

The usage of Mashavu by CHWs suggests that a large population is currently unaware of the personal health challenges that they face. Through preventive health services, communities have received personal health knowledge and can make strides towards early treatment. By improving this knowledge in individuals through the $\mathrm{CHW}$ provided information, an existing barrier of health access in vulnerable communities was overcome (Ensor \& Cooper, 2004).

\section{CONCLUSION AND RECOMMENDATIONS}

In order for health services such as Mashavu to succeed, it is critical to build confidence among consumers in the system's value and maintain CHW involvement. Over the course of the pilot, the CHWs learned that people in their rural communities were willing to pay for preventive health services. This pilot successfully achieved the CHW buy-in that is a necessary prerequisite for the transition to an unsubsidised business model, in which the CHWs pay Mashavu for any and all supplies provided.

The implementation of a complex telemedicine system led to notoriety within the communities and influential socio-political circles. The complexity inherent to a system with multiple inputs necessitated a high level of resources and accountability on the part of all of those that were involved. Notoriety helped the pilot to gain momentum and inspire the CHWs to continue working.

After the five-month pilot completed, the CHWs gained increased autonomy and sought to streamline operations. The added cost and complexity of providing a full consultation prohibited economic viability. To ensure long-term success, economically and operationally, Mashavu opted to pursue a model that did not rely on computers or full-time nurses. The CHWs continued by offering only the body measurements and general health advice, allowing them to conduct mobile operations and mitigate costs. A new model was effectively started in which CHWs pursued work independently on foot while carrying carbon copy receipt books and a backpack with only the necessary medical devices. The CHWs experienced a high volume of clients allowing them to generate profit daily and not rely on subsidies. This stable income enabled the CHWs to pay a portion of their wages to Mashavu to work towards ownership of the medical devices. This model now engages six MHWs that provide 'know your numbers' service in the Nyeri area with a cash-positive revenue model. Comparative analysis of this new model is the subject of future research.

\section{REFERENCES}

Bolin, T., 1996, 'Cost benefit of early diagnosis of colorectal cancer'. Scandinavian Journal of Gastroenterology, vol. 31, no. S220, pp. 142-146.

Briscombe, B., Sharma, S. \& Saunders, M., 2010, Improving resource allocation in Kenya's public health sector, Washington DC: Futures Group, Health Policy Initiative, Task Order 1.

Castro-Leal, F., Dayton, J., Demery, L. \& Mehra, K., 2000, 'Public spending on health care in Africa: do the poor benefit?', Bulletin of the World Health Organization,vol. 78, no. 1, pp. 66-72. 
Chen, S., Cheng, A. \& Mehta, K., 2013, 'A Review of Telemedicine Business Models', Telemedicine and e-Health, April, vol. 19, no. 4, pp. 287-297.

Chuma, J., Gilson, L. \& Molyneux, C., 2007, 'Treatment-seeking behaviour, cost burdens and coping strategies among rural and urban house holds in coastal Kenya: an equity analysis', Journal of Tropical Medicine E International Health, vol. 12, no. 5, pp. 673-686.

Ensor, T. \& Cooper, S., 2004, 'Overcoming barriers to health service access: influencing the demand side, Health Policy and Planning, vol. 19, no. 2, pp. 69-79.

Fleishman, A., Fleishman, A.J., Wittig, J., Milnes J., Baxter, A., Moreau, J., Mehta, K., 2010, 'Validation process for a social entrepreneurial telemedicine venture in East Africa', International Journal for Service Learning Engineering, vol. 5, no. 1, pp. 1-24.

Food and Agriculture Organization, 1996, Rome Declaration on World Food Security and World Food Summit Plan of Action, viewed 29 July 2012, <http: / / www.fao.org/docrep/003/w3613e/w3613e00.htm>.

Google Maps, 2014. Map. Nyeri: Google Maps.

GSMA, 2012. Safaricom - Kenya - Feasibility Study, GMSA Head Office, London, UK.

Gu, W., Wang, X. \& McGregor, S. E., 2010, 'Optimization of preventive health care facility locations', International Journal of Health Geographics, vol. 9, no. 17, pp. 1-16.

Holmes, K., Suffian, S., Lackey, J., Mehta, K., 'Pilot Results of a Health Social Franchise in Kenya: Early Evidence of Sustainable Livelihood Creation', paper presented to Humanitarian Technology: Science, Systems and Global Impact Conference, Boston, May 2014

Kelly, J. M., Osamba, B., Garg, R.M., Hamel, M.J., Lewis, J.J., Rowe, S.Y., Rowe, A.K., Deming, M.S.,
2001, 'Community Health Worker Performance in the Management of Multiple Childhood Illnesses: Siaya District, Kenya, 1997-2001' , American Journal of Public Health, October, vol. 91, no.10, pp. 1617-1624.

Ochako, R., Fotso, J.C., Ikamari, L., Khasakhala, A., 2011, 'Utilization of maternal health services among young women in Kenya: Insights from the Kenya Demographic and Health Survey, 2003', BMC Pregnancy and Childbirth, vol. 11 no. 1, pg. 1.

Pandya, R., Grillo, D., Lycksell, E., Mieybegue, P., 1997, 'IMT-2000 Standards: Network Aspects', IEEE Personal Communications, August, IEEE vol. 4, no. 4, pp. 20-29.

Qin, R., Dzombak, R., Amin, R. \& Mehta, K., 2012, 'Reliability of a telemedicine system designed for rural Kenya', Journal of Primary Care and Community Health, vol. 4 , no. 3, pp. 1-5.

Wachira, B. \& Martin, I. B., 2011, 'The state of emergency care in the Republic of Kenya', African Journal of Emerging Medicine, vol. 1, no. 4, pp. 160-165.

Wang, L., Kong, L., Wu, F., Yamin, B., Burton, R., 2005, 'Preventing chronic diseases in China', Lancet, vol. 366, no. 9499, pp. 1821-1824.

World Health Organization, 2009, Country Statistics, Kenya, World Health Organization, Geneva, Switzerland.

World Health Organization, 2011, World Health Statistics, World Health Organization, Geneva, Switzerland.

Wynchak, S. \& Fortuin, J., 2012, 'Africa's telenursing today (and tomorrow?)', International Journal of Advance Life Sciences, vol. 2, no. 3 and 4, pp. 165-172.

Yonga, G., 2010, Case Kenya Study: NCD Situation, Workshop on Country-Level Decision Making for Control of Chronic Diseases. Washington, DC: Institute of Medicine of the National Academies. 


\section{APPENDICES}

Appendix A: Demographic information screenshot.

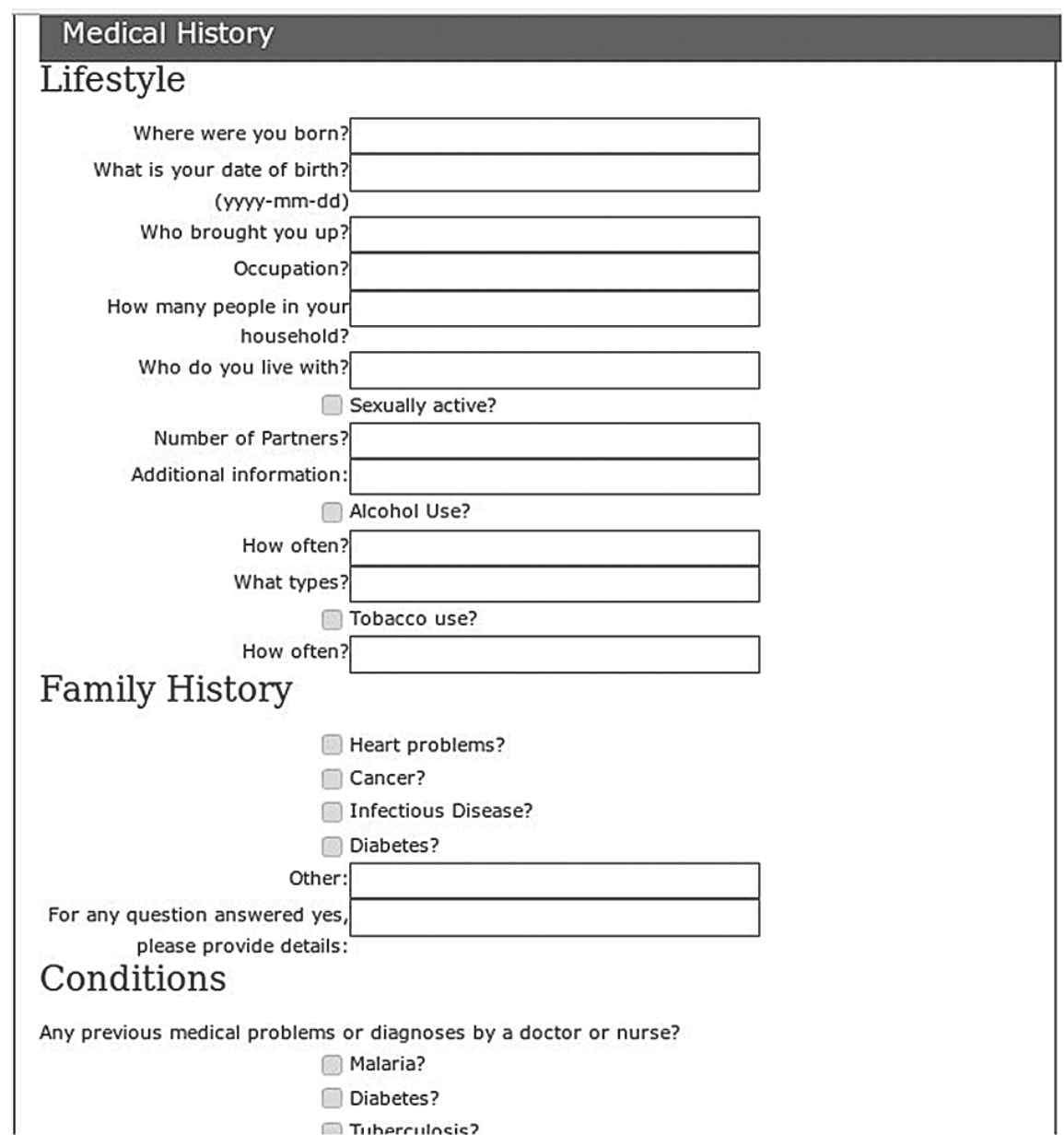

Appendix B: Consultation questions screenshot.

\begin{tabular}{|c|c|c|}
\hline New Patients & Consultation Questions & \\
\hline Patient Lookup & \multicolumn{2}{|l|}{ Upload } \\
\hline New Responses (0) & test & Patient ID: 7 \\
\hline Pending Responses ( 0 ) & \multirow{3}{*}{$\begin{array}{l}\text { Gender: | Date Of Birth: } \\
\text { City: | Country: } \\
\text { Phone\#: }\end{array}$} & \\
\hline Kiosk Statistics & & \\
\hline & & \\
\hline
\end{tabular}

General

What are your top 2 health
complaints?

Have you been treated by a traditional healer for anything?

What medicines did the traditional healer provide?

Ears/Nose/Throat Have you had any:

$\square$ Change in vision (sight problems)?

$\square$ Eye pain (eyes hurting)?

Decreased hearing (listening problems)?

$\square$ Pain in your ears (ears hurting)?

Discharge from the ears? 